Tropical Journal of Pharmaceutical Research April 2021; 20 (4): 695-701

ISSN: $1596-5996$ (print); 1596-9827 (electronic)

(C) Pharmacotherapy Group, Faculty of Pharmacy, University of Benin, Benin City, 300001 Nigeria.

\title{
Microglia inflammatory response contributes to chronic constriction injury-induced neuropathic pain via miR- 339/PFKFB3 axis
}

\author{
Xiaolian Fan ${ }^{1}$, Renqing Zeng ${ }^{2}$, Longxue Cai ${ }^{2 *}$ \\ ${ }^{1}$ Department of Neurology, Huanggang Central Hospital of Hubei Province, Huanggang City, Hubei Province 438000, \\ ${ }^{2}$ Department of Emergency, First Affiliated Hospital of Gannan Medical University, Ganzhou City, Jiangxi Province 341000, \\ China
}

*For correspondence: Email: Ixcai999@163.com; Tel: +86-797-8266028

Sent for review: 3 December 2020

Revised accepted: 25 March 2021

\begin{abstract}
Purpose: To investigate the effect of miR-339 on neuropathic pain.

Methods: A rat neuropathic pain model was established through chronic constriction injury (CCl). Expression of miR-339 in spinal cord was determined 14 days later. Microglial inflammatory response was evaluated using immunofluorescence analysis of ionized calcium binding adaptor molecule 1 (Iba1), while IL-6 and TNF- $\alpha$ were assessed by enzyme-linked immunosorbent assay (ELISA). Painassociated behavioral effects and microglia-related inflammation were investigated after intrathecal administration of miR-339 agomir into rats post-CCl. The target gene of miR-339 involved in neuropathic pain was evaluated by a luciferase reporter assay. Microglia cells were isolated from rats, then treated with lipopolysaccharide (LPS). The LPS-induced inflammatory response in microglia cells was determined using quantitative reverse transcription PCR analysis of IL-6 and TNF- $\alpha$.

Results: CCl decreased mechanical allodynia and thermal hyperalgesia thresholds, but increased Iba1, IL-6, and TNF- $\alpha$ in rats. MiR-339 was reduced in rat spinal cord after $\mathrm{CCl}$ induction while intrathecal injection of miR-339 agomir alleviated CCl-induced changes in mechanical and thermal hyperalgesia in rats, and reversed expression of Iba1, IL-6, and TNF-a. Furthermore, 6-phosphofructo-2kinase/fructose-2,6-bisphosphatase-3 (PFKFB3) was identified as a miR-339 target gene, and overexpression of miR-339 decreased the expression of PFKFB3, IL-6, and TNF- $\alpha$ in LPS-induced microglia cells.

Conclusion: The miR-339/PFKFB3 axis ameliorates CCl-induced neuropathic pain by suppression of microglia inflammatory response, suggesting a novel strategy for neuropathic pain management.
\end{abstract}

Keywords: Neuropathic pain, miR-339, Chronic constriction injury, PFKFB3, Microglia cells

This is an Open Access article that uses a fund-ing model which does not charge readers or their institutions for access and distributed under the terms of the Creative Commons Attribution License (http://creativecommons.org/licenses/by/4.0) and the Budapest Open Access Initiative (http://www.budapestopenaccessinitiative.org/read), which permit unrestricted use, distribution, and reproduction in any medium, provided the original work is properly credited.

Tropical Journal of Pharmaceutical Research is indexed by Science Citation Index (SciSearch), Scopus, International Pharmaceutical Abstract, Chemical Abstracts, Embase, Index Copernicus, EBSCO, African Index Medicus, JournalSeek, Journal Citation Reports/Science Edition, Directory of Open Access Journals (DOAJ), African Journal Online, Bioline International, Open-J-Gate and Pharmacy Abstracts

\section{INTRODUCTION}

Neuropathic pain is a pain syndrome caused by primary lesions or dysfunction of the central or peripheral nervous system [1]. Tactile pain is a major feature of neuropathic pain, mainly manifested as harmless tactile stimulation that induces a pain response [1]. Due to the 
complicated pathogenesis of neuropathic pain, current effective treatment strategies are very limited [2]. Therefore, development of optimal strategies to manage neuropathic pain is an urgent need.

Microglia cells, with the ability to regulate synaptic remodeling and immune surveillance in the central nervous system, underlie neuropathic pain [3]. Drugs targeting microglia might offer potential therapeutic strategies for neuropathic pain [3]. In response to noxious stimuli, microglia cells release inflammatory factors, such as TNF$\alpha$ and $\mathrm{IL}-1 \beta$, and contribute to pain hypersensitivity during neuroimmune activation of neuropathic pain [4]. Administration of microglia inhibitor attenuates pain hypersensitivity in neuropathic pain [4]. Therefore, regulators of the microglial inflammatory response could be effective as antinociceptive agents in neuropathic pain management.

MicroRNA (miRNA) plays key roles in immune cell differentiation and activation [5]. Dysregulated miRNA expression has been implicated in the microglia-mediated inflammatory response, thus participating in the pathogenesis of neuropathic pain [5]. A recent study showed that the expression of miRNAs in the nervous system regulates neuroimmunity and neuropathic pain [6]. MiR-339 was found to be reduced in neuropathic pain in rats [7], and demonstrated anti-inflammatory activity in alcohol-exposed brain tissues and microglia cells [8]. However, no relevant studies of mechanisms involved in miR-339-mediated neuropathic pain have yet been reported. Therefore, the present study established a rat model of neuropathic pain through chronic constriction injury $(\mathrm{CCl})$, and performed functional assays to evaluate effects of miR-339 on neuropathic pain. Microglia cells were isolated, and the role and mechanism of miR-339 on the microglial inflammatory response were determined.

\section{EXPERIMENTAL}

\section{Rat model of neuropathic pain}

The study was approved by the Scientific Research Ethics Committee of First Affiliated Hospital of Gannan Medical University (approval no. LLSC-2020061901), and conducted in accordance with the National Institutes of Health Laboratory Animal Care and Use Guidelines [9]. Eighteen male Wistar rats (13 weeks old, 300 $350 \mathrm{~g}$ ), acquired from Shanghai Animal Laboratory Center (Shanghai, China), were divided into three groups: sham, $\mathrm{CCl}+\mathrm{NC}$ agomir, and $\mathrm{CCl}+\mathrm{miR}-339$ agomir. For the $\mathrm{CCl}$ model, rats were anesthetized using intraperitoneal injection of $60 \mathrm{mg} / \mathrm{kg}$ sodium pentobarbital. The left sciatic nerve was then exposed and ligated with $4 / 0$ silk, proximal to the trifurcation and $1 \mathrm{~mm}$ from the four ligations. Ethicon silk sutures were then used to suture the muscle and skin layers. Rats in the sham group underwent the same procedure without ligation of the left sciatic nerve. Pain-associated behavioral activities were investigated $1,4,7$, and 14 days after the surgery.

For drug infusion, rats with $\mathrm{CCl}$ induction were anesthetized, and lumbar laminectomy of the L5 vertebra was performed. Following cutting of the dura, a polyethylene-10 catheter was inserted into the subarachnoid space at $L 4 / 5$ of the spinal cord. After lumbar implantation of the intrathecal catheter, the rats were allowed to recover for 3 days before drug delivery. Four days after $\mathrm{CCl}$ surgery, 5 nM miR-339 agomir or NC agomir (20 $\mu \mathrm{L}$; Ribobio, Beijing, China) were intrathecally administered into the catheter through a connected microinjection syringe. The drugs were administered once a day from day 4 to day 8. Pain-associated behavioral activities were also investigated $1,4,7$, and 14 days after the drug delivery.

\section{Behavioral test}

Rats were habituated in a wire mesh, and an automated dynamic plantar aesthesiometer (Ugo Basile, Varese, Italy) was used to measure mechanical allodynia. The mechanical withdrawal threshold (MWT) was calculated as the lowest force $(\mathrm{g})$ leading to rapid withdrawal of the right hind paw. Each measurement was repeated three times to determine the average force. For thermal hyperalgesia, rats were habituated in a clear glass plate at $30{ }^{\circ} \mathrm{C}$. A heat source was then positioned beneath the hind paw. Paw withdrawal thermal latency (PWTL) was calculated as the duration (s) between the start of heat application and withdrawal of the paw, measured with a digital timer. Each measurement was also repeated three times to determine the average time.

\section{Enzyme-linked immunosorbent assay (ELISA)}

Fourteen days after $\mathrm{CCl}$ surgery and drug delivery, spinal cord segments L4-L6 were harvested, incubated with RIPA cell lysis buffer (Beyotime Institute of Biotechnology, Shanghai, China), and the supernatants were collected after centrifugation. Levels of TNF- $\alpha$ and IL- 6 were determined using ELISA kits (Abcam, Cambridge, MA, USA). 


\section{Immunofluorescence}

Fourteen days after $\mathrm{CCl}$ surgery and drug delivery, the transverse spinal cords were collected and cut into $25 \mu \mathrm{m}$-thick sections. After fixation with $4 \%$ paraformaldehyde and blocking with horse serum (Jackson ImmunoResearch, West Grove, PA, USA), the sections were incubated with anti-lba1 antibody (1:200, Abcam) at $4{ }^{\circ} \mathrm{C}$ overnight. Following staining with Alexa Fluor $®$ 488-conjugated secondary antibody (1:500, Abcam), images were acquired with an FV1000 fluorescence microscope (Olympus, Tokyo, Japan).

\section{Microglia cell isolation}

Brain tissues were collected from rats, and then were dissociated to generate mixed primary glia cell culture. Microglia cells were isolated from the mixture by mild trypsinization using $0.05 \%$ trypsin, and then cultured in DMEM (Dulbecco's Modified Eagle Medium) containing 10\% fetal bovine serum. HEK293T cells (ATCC, Manassas, VA, USA) were also grown in DMEM at $37^{\circ} \mathrm{C}$ in a humidified incubator.

\section{Cell transfection and treatment}

Microglia cells were transfected with miR-339 mimic or NC mimic (Ribobio) via Lipofectamine ${ }^{\mathrm{TM}}$ 2000 (Invitrogen, Waltham, MA, USA). HEK293T cells were also transfected with miR-339 mimic or inhibitor, or with NC mimic or inhibitor. For LPS treatment, microglia cells, with or without transfection, were incubated with DMEM containing $5 \mu \mathrm{g} / \mathrm{mL}$ LPS for $24 \mathrm{~h}$.

\section{Luciferase reporter assay}

Sequences of wild type or mutant PFKFB3 3' UTR were cloned into pMIR-GLO ${ }^{\text {TM }}$ Luciferase vector (Promega, Madison, WI, USA). HEK293T cells were co-transfected with PFKFB3-WT or PFKFB3-MUT luciferase vector and miR-339 mimic or NC mimic. Forty-eight hours later, DualLuciferase Reporter Assay system (Promega) was used to measure luciferase activity.

\section{Quantitative reverse transcription PCR (qRT- PCR)}

RNA was extracted from HEK293T cells, microglia cells, or dorsal horn of rat spinal cord via TRIzol reagent (Invitrogen), and reversetranscribed into cDNA with the MMLV RT kit (Promega). Relative expression of miR-339 and PFKFB3 were determined using TaqMan microRNA assays (Thermo Fisher), using the primers indicated in Table 1.
Table 1: Primer sequences

\begin{tabular}{|c|c|}
\hline ID & Sequence (5'- 3') \\
\hline MiR-339 F & $\begin{array}{l}\text { CGACGCGTCGCGCCATTGCCAC } \\
\text { GGCACCAT }\end{array}$ \\
\hline MiR-339 R & $\begin{array}{l}\text { CCATCGATGGGGCAGAAGACCC } \\
\text { ACGCATACGAGT }\end{array}$ \\
\hline $\begin{array}{l}\text { PFKFB3 F } \\
\text { PFKFB3 R }\end{array}$ & $\begin{array}{l}\text { CCTCTGCTTTTGAAACAGGC } \\
\text { ATCCAGACAGACACTGCACG }\end{array}$ \\
\hline IL-6 F & $\begin{array}{l}\text { TGGAGTCACAGAAGGAGTGGCT } \\
\text { AAG }\end{array}$ \\
\hline IL-6 R & $\begin{array}{l}\text { TCTGACCACAGTGAGGAATGTC } \\
\text { CAC }\end{array}$ \\
\hline TNF- $\alpha \mathrm{F}$ & CСССТCAGCAAACCACCAAGT \\
\hline TNF- $\alpha \mathrm{R}$ & CTTGGGCAGATTGACCTCAGC \\
\hline$\beta$-actin F & CCACACCCGCCACCAGTTCG \\
\hline$\beta$-actin R & СССАТТСССАССАТСАСАСС \\
\hline U6 F & GCTCGCTTCGGCAGCACA \\
\hline U6 R & GAGGTATTCGCACCAGAGGA \\
\hline
\end{tabular}

\section{Western blot assay}

Proteins were extracted from HEK293T and microglia cells via RIPA lysis buffer, and quantified by bicinchoninic acid assay (Beyotime). Protein samples $(30 \mu g)$ were separated by sodium dodecyl sulphatepolyacrylamide gel electrophoresis, then transferred onto a polyvinylidene difluoride membrane (EMD Millipore, Bedford, MA, USA). After blocking in 5\% non-fat dry milk, membranes were incubated with primary antibodies against PFKFB3 (1:2000; Abcam) or $\beta$-actin (1:3000; Abcam). Following incubation with horseradish peroxidase-conjugated secondary antibodies (1:5000), protein signals were visualized using the Super Signal West Pico Chemiluminescent Substrate kit (Thermo Fisher; Waltham, MA, USA).

\section{Statistical analysis}

Data are expressed as mean \pm standard deviation. GraphPad Prism 6.0 was used to conduct statistical analyses using Student's $t$-test or one-way analysis of variance. Values of $p<$ 0.05 were considered statistically significant.

\section{RESULTS}

\section{MiR-339 was reduced in dorsal horn of spinal cord in $\mathrm{CCl}$ rats}

To establish the neuropathic pain model, rats were subjected to $\mathrm{CCl}$ induction. Analysis of data for mechanical allodynia and thermal hyperalgesia showed that $\mathrm{CCl}$ could decrease MWT (Figure $1 \mathrm{~A}$ ) and PWTL (Figure $1 \mathrm{~B}$ ) measured $1,4,7$, or 14 days post-CCl, suggesting successful establishment of the neuropathic pain model. Expression of miR-339 was reduced in the dorsal horn of the spinal cord 
in $\mathrm{CCl}$ rats compared to the sham group (Figure $1 \mathrm{C})$. Higher levels of IL-6 and TNF- $\alpha$ demonstrated that $\mathrm{CCl}$ induced neuroinflammatory processes (Figure 1 D). Expression of lba1, a marker of activated microglial cells, was also up-regulated in rats post-CCl (Figure $1 \mathrm{E}$ ), suggesting that $\mathrm{CCl}$ induces a microglial inflammatory response in rats.

A

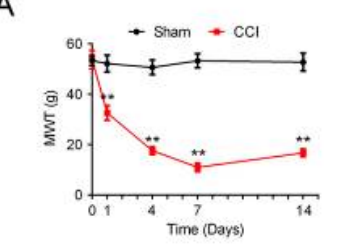

C

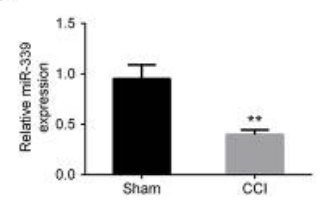

E

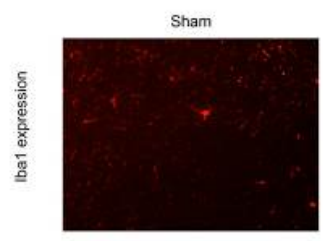

Figure 1: MiR-339 was reduced in dorsal horn of spinal cord in $\mathrm{CCl}$ rats. (A) Mechanical allodynia analysis of rats post-CCl establishment. (B) Thermal hyperalgesia analysis of rats post-CCl establishment. (C) Expression of miR-339 in dorsal horn of spinal cord in $\mathrm{CCl}$ rats. (D) Expressions of IL-6 and TNF- $\alpha$ in dorsal horn of spinal cord in $\mathrm{CCl}$ rats. (E) Expression of Iba1 in dorsal horn of spinal cord in $\mathrm{CCl}$ rats; ${ }^{* *} p<$ 0.01 vs. sham

\section{Forced miR-339 expression attenuated neuropathic pain development}

To investigate the functional role of miR-339 in neuropathic pain, rats in which $\mathrm{CCl}$ had been induced were injected intrathecally with miR-339 agomir. Mechanical allodynia (Figure $2 \mathrm{~A}$ ) and thermal hyperalgesia (Figure $2 \mathrm{~B}$ ) were reduced by injection of miR-339 agomir, compared to NC agomir, suggesting that miR-339 alleviates neuropathic pain. Rats injected with miR-339 agomir exhibited increased miR-339 expression (Figure $2 \mathrm{C}$ ), and decreased IL-6 and TNF- $\alpha$ secretion (Figure 2 D). Moreover, expression of Iba1 protein was reduced in $\mathrm{CCl}$ rats injected with miR-339 agomir (Figure 2 E), suggesting that forced miR-339 expression may attenuate neuropathic pain development through inhibition of the microglial inflammatory response.
A

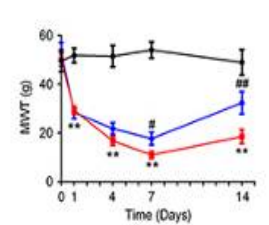

C

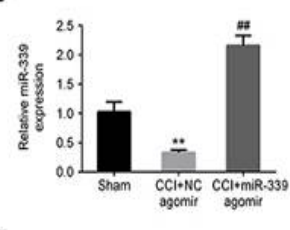

D
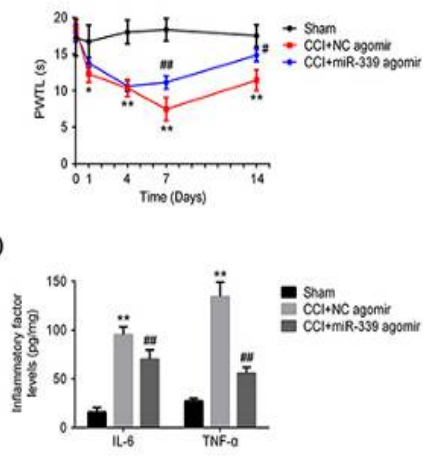

$\mathrm{E}$
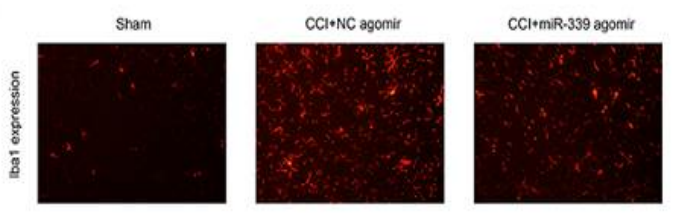

Figure 2: Forced miR-339 expression attenuated neuropathic pain development. (A) Mechanical allodynia of rats injected intrathecally with miR-339 agomir or NC agomir. (B) Thermal hyperalgesia analysis of rats injected intrathecally with miR-339 agomir or NC agomir. (C) Expression of miR-339 in dorsal horn of spinal cord in $\mathrm{CCl}$-induced rats injected intrathecally with miR-339 agomir or NC agomir. (D) Expression of IL-6 and TNF- $\alpha$ in dorsal horn of spinal cord in $\mathrm{CCl}$-induced rats injected intrathecally with miR-339 agomir or NC agomir. (E) Expression of Iba1 in dorsal horn of spinal cord in $\mathrm{CCl}$-induced rats injected intrathecally with miR-339 agomir or NC agomir. \# and \#\# denote $p<0.05$ and $p<0.01$ vs. CCl $+\mathrm{NC}$ agomir, respectively; ${ }^{* *} p<0.0$ vs. sham

\section{PFKFB3 was targeted by miR-339}

PFKFB3, containing a potential binding site for miR-339, was then identified as a target gene of miR-339 (Figure 3 A). Luciferase activity of PFKFB3-WT was decreased by transfection with miR-339 mimic, compared to NC mimic (Figure 3 $B)$. Moreover, mutation of the miR-339 binding site in PFKFB3 abolished the suppressive effect of miR-339 on luciferase activity (Figure $3 \mathrm{~B}$ ), suggesting that miR-339 binds to PFKFB3. To further investigate regulation of PFKFB3 by miR339, HEK293T cells were transfected with miR339 mimic or inhibitor, and the expression of PFKFB3 was then evaluated via qRT-PCR and western blot. Results showed that miR-339 decreased mRNA (Figure $3 \mathrm{D}$ ) and protein (Figure $3 \mathrm{E}$ ) expression of PFKFB3, while transfection with miR-339 inhibitor enhanced the expression of PFKFB3 (Figure $3 \mathrm{D}$ and $\mathrm{E}$ ). These results showed that miR-339 targeted PFKFB3. 
A

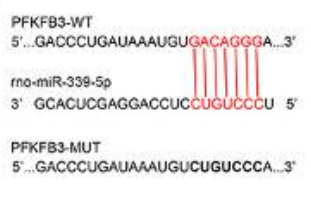

C

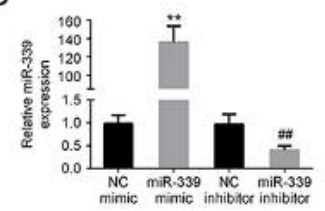

E

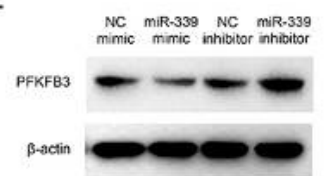

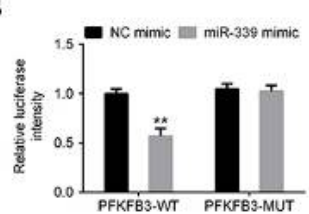

D
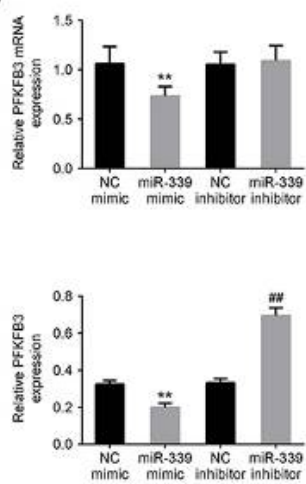

Figure 3: PFKFB3 was targeted by miR-339. Potential binding site between miR-339 and PFKFB3. (B) Effect of miR-339 on luciferase activities of PFKFB3-WT and PFKFB3-MUT. (C) Transfection efficiency of miR-339 mimic and inhibitor in HEK293T cells. (D) Effect of miR-339 on mRNA expression of PFKFB3. (E) Effect of miR-339 on protein expression of PFKFB3. **, \#\# $p$ $<0.01$ vs. NC mimic or vs. NC inhibitor, respectively

Forced miR-339 expression suppressed LPSinduced inflammation in microglia cells

Primary microglial cells were isolated and induced with LPS, to evaluate the role of miR339 on microglia inflammation in vitro. LPS treatment stimulated increased levels of IL-6 and TNF- $\alpha$ (Figure $4 \mathrm{~A}$ ), while transfection with miR339 mimic reduced LPS-induced increases in IL6 and TNF- $\alpha$ (Figure 4 A), suggesting that forced miR-339 expression suppressed LPS-induced inflammation in microglia cells. Moreover, expression of PFKFB3 protein was enhanced in microglia cells after LPS treatment (Figure 4 B), but the response was reduced by miR-339 mimic transfection (Figure $4 \mathrm{~B}$ ), indicating that miR-339 may regulate the microglial inflammatory response through targeting of PFKFB3.

\section{DISCUSSION}

Previous research has shown that an inflammatory response in the central nervous system, modulated by activated microglia cells, results in neuronal cell death and aggravates neurodegenerative diseases, including neuropathic pain [10]. Therefore, miRNAs with the ability to regulate the microglia-mediated immune response [5] might provide a promising approach to ameliorate neuropathic pain [11].

A

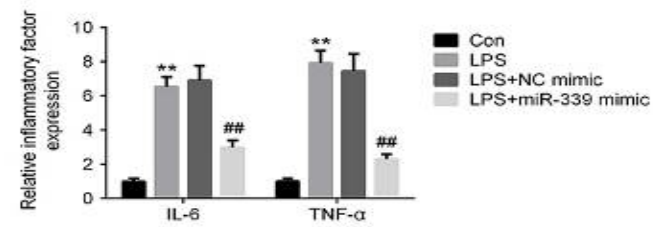

B
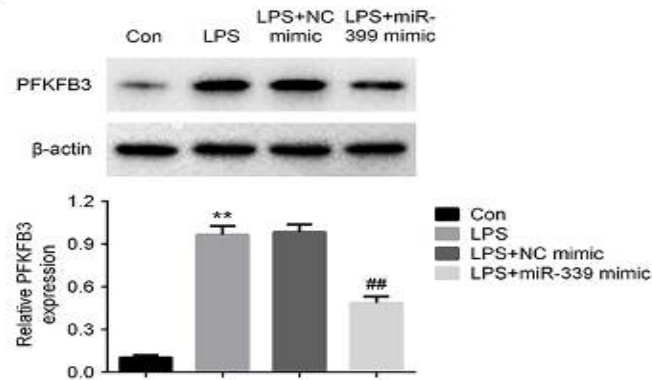

Figure 4: Forced miR-339 expression suppressed LPS-induced inflammation in microglia cells. (A) Expression of IL-6 and TNF- $\alpha$ in LPS-induced microglia cells, with or without transfection of miR-339 mimic. (B) Protein expression of PFKFB3 in LPSinduced microglia cells, with or without transfection of miR-339 mimic. ${ }^{* *}$ \#\#p $<0.01$ vs. control or vs. LPS + NC mimic, respectively

Because miR-339 suppressed inflammation in alcohol-induced microglia cells [8], favorable effects of miR-339 on the microglia inflammatory response in neuropathic pain were investigated in this study.

Ligation of the left sciatic nerve in the $\mathrm{CCl}$ model can lead to inflammation and swelling, thus resulting in nociceptive outcomes with decreased MWT and PWTL [12]. Therefore, $\mathrm{CCl}$ induction has been widely used as a neuropathic pain model. In addition to nociceptive outcomes, $\mathrm{CCl}$ also induces neuroinflammation with increased inflammatory cytokines, including TNF- $\alpha$ and IL-6 [13]. This study successfully established the CClinduced neuropathic model, with reduced thresholds for mechanical allodynia and thermal hyperalgesia as well as increased TNF- $\alpha$ and IL6. Moreover, activation of microglia cells is related to inflammation in neuropathic pain [14]. Microglia activation could induce neuronal damage during neuropathic pain through release of inflammatory cytokines [10]. The biomarker of microglia cell activation, Iba1, was also upregulated post-CCl establishment, suggesting that $\mathrm{CCl}$ induction promotes microglia activation in neuropathic pain.

A previous study has shown that miR-339 was reduced in dorsal root ganglion of rats following spinal nerve ligation [7]. The present study also indicated a significant decrease of miR-339 in 
dorsal horn of the spinal cord in $\mathrm{CCl}$ rats. Functional assays further revealed that intrathecal injection of miR-339 agomir exerted anti-nociceptive and anti-inflammatory roles in $\mathrm{CCl}$ rats, resulting in increased MWT and PWTL, and decreased TNF- $\alpha$ and IL-6. Moreover, suppression of microglia activation has been regarded as a potential therapeutic strategy for neurodegenerative disease [10]. Activation of microglia cells was suppressed by intrathecal injection of miR-339 agomir, suggesting that miR-339 attenuates the microglia inflammatory response in $\mathrm{CCI}$ rats. An in vitro neuropathic pain model, established by isolated microglia cells treated with LPS [15], also showed increased inflammatory cytokines, TNF- $\alpha$ and IL-6. Forced miR-339 expression also decreases TNF- $\alpha$ and IL-6 and suppresses LPS-induced inflammation in microglia cells, confirming the antiinflammatory role of miR-339 in microglia cells.

I-kappa B kinase was validated as a miR-339 binding target in alcohol-induced brain inflammation [8]. In this study, PFKFB3 was determined as a target gene of miR-339. Previous study has shown that LPS promotes an inflammatory state, with increased PFKFB3, in microglia cells [16]. Therefore, PFKFB3 was implicated in microglial inflammation. Data obtained in the present study showed that LPS treatment increased the PFKFB3 level in microglia cells, and promoted microglial inflammation, with enhanced TNF- $\alpha$ and IL-6. Over-expression of PFKFB3 has been shown to increase IL-1 $\beta$ and IL- 6 to stimulate microglial polarization in the pro-inflammatory M1 phenotype [17]. Switching of microglial polarization from $\mathrm{M} 1$ to an anti-inflammatory $\mathrm{M} 2$ phenotype could suppress CCl-induced neuropathic pain [18]. This study showed that forced miR-339 expression decreased the LPSinduced up-regulation of PFKFB3, attenuating microglial inflammation. However, the role of miR-339 in microglial polarization should be investigated further. Furthermore, intrathecal injection of lentiviruses expressing siRNA to target PFKFB3 expression in $\mathrm{CCl}$-induced rats should also be performed to elucidate the functional role of miR-339/PFKFB3 in neuropathic pain.

\section{CONCLUSION}

MiR-339 suppressed the microglial inflammatory response to ameliorate neuropathic pain through down-regulation of PFKFB3. The miR339/PFKFB3 axis might be a promising therapeutic strategy for the treatment of neuropathic pain.

\section{DECLARATIONS}

\section{Conflict of interest}

No conflict of interest is associated with this work.

\section{Contribution of authors}

We declare that this work was done by the authors named in this article and all liabilities pertaining to claims relating to the content of this article will be borne by the authors. Xiaolian Fan and Renqing Zeng designed the study, supervised the data collection, and analyzed the data. Longxue Cai interpreted the data, prepared the manuscript for publication, and reviewed the draft of the manuscript. All authors have read and approved the manuscript.

\section{Open Access}

This is an Open Access article that uses a funding model which does not charge readers or their institutions for access and distributed under the terms of the Creative Commons Attribution License (http://creativecommons.org/licenses/by/ 4.0) and the Budapest Open Access Initiative (http://www.budapestopenaccessinitiative.org/rea d), which permit unrestricted use, distribution, and reproduction in any medium, provided the original work is properly credited.

\section{REFERENCES}

1. $X u W$, Zhu $X$, Zhan $G$, Sheng $L$, Chen $Y$. Vitexin alleviates neuropathic pain in a mouse chronic constriction injury model by inactivation of NF-KB. Tro $J$ Pharm Res 2020; 19(5): 989-994.

2. Lee GH, Kim SS. Therapeutic Strategies for Neuropathic Pain: Potential Application of Pharmacosynthetics and Optogenetics. Mediat Inflamm 2016; 2016: 58082155808215.

3. Inoue K, Tsuda M. Microglia in neuropathic pain: cellular and molecular mechanisms and therapeutic potential. Nat Rev Neurosci 2018; 19(3): 138-152.

4. Carniglia L, Ramírez D, Durand D, Saba J, Turati J, Caruso C, Scimonelli TN, Lasaga M. Neuropeptides and Microglial Activation in Inflammation, Pain, and Neurodegenerative Diseases. Mediat Inflamm 2017; 2017: 5048616-5048616.

5. Guedes J, Cardoso AL, Pedroso de Lima MC. Involvement of microRNA in microglia-mediated immune response. Clin Dev Immunol 2013; 2013: 186872.

6. Imai S, Saeki M, Yanase $M$, Horiuchi $H$, Abe $M$, Narita $M$, Kuzumaki N, Suzuki T, Narita M. Change in microRNAs associated with neuronal adaptive responses in the 
nucleus accumbens under neuropathic pain. J Neurosci 2011; 31(43): 15294-15299.

7. von Schack D, Agostino MJ, Murray BS, Li Y, Reddy PS, Chen J, Choe SE, Strassle BW, Li C, Bates B et al. Dynamic changes in the microRNA expression profile reveal multiple regulatory mechanisms in the spinal nerve ligation model of neuropathic pain. PloS one 2011; 6(3): e17670-e17670.

8. Zhang Y, Wei G, Di Z, Zhao Q. miR-339-5p inhibits alcohol-induced brain inflammation through regulating NF-kappaB pathway. Biochem Biophys Res Commun 2014; 452(3): 450-456.

9. Care IoLARCo, Animals UoL: Guide for the care and use of laboratory animals: US Department of Health and Human Services, Public Health Service, National; 1986.

10. Dheen ST, Kaur C, Ling EA. Microglial activation and its implications in the brain diseases. Curr Med Chem 2007; 14(11): 1189-1197.

11. Willemen HLDM, Huo $X-J$, Mao-Ying Q-L, Zijlstra J, Heijnen CJ, Kavelaars A. MicroRNA-124 as a novel treatment for persistent hyperalgesia. J Neuroinflamm 2012; 9: 143-143.

12. Huang C-P, Lin $Y-W$, Lee $D-Y$, Hsieh C-L. Electroacupuncture Relieves CCI-Induced Neuropathic Pain Involving Excitatory and Inhibitory Neurotransmitters. Evid-Based Compl Alt: eCAM 2019; 2019: 6784735-6784735.
13. Wang C, Wang C. Anti-nociceptive and anti-inflammatory actions of sulforaphane in chronic constriction injuryinduced neuropathic pain mice. Inflammopharmacology 2017; 25(1): 99-106.

14. Ni HD, Yao M, Huang $B, X u L S$, Zheng $Y$, Chu YX, Wang $H Q$, Liu MJ, Xu SJ, Li HB. Glial activation in the periaqueductal gray promotes descending facilitation of neuropathic pain through the p38 MAPK signaling pathway. J Neurosci Res 2016; 94(1): 50-61.

15. Jin G-L, He S-D, Lin S-M, Hong $L-M$, Chen $W-Q, X u Y$, Yang J, Li S-P, Yu C-X. Koumine Attenuates Neuroglia Activation and Inflammatory Response to Neuropathic Pain. Neural Plast 2018; 2018: 9347696-9347696.

16. Rubio-Araiz A, Finucane OM, Keogh S, Lynch MA. AntiTLR2 antibody triggers oxidative phosphorylation in microglia and increases phagocytosis of beta-amyloid. J Neuroinflamm 2018; 15(1): 247.

17. Kong L, Wang Z, Liang X, Wang Y, Gao L, Ma C. Monocarboxylate transporter 1 promotes classical microglial activation and pro-inflammatory effect via 6phosphofructo-2-kinase/fructose-2, 6-biphosphatase 3. J Neuroinflamm 2019; 16(1): 240.

18. Gui $X$, Wang $H$, Wu L, Tian S, Wang $X$, Zheng $H$, Wu W. Botulinum toxin type $A$ promotes microglial $M 2$ polarization and suppresses chronic constriction injuryinduced neuropathic pain through the $P 2 X 7$ receptor. Cell Biosci 2020; 10: 45-45. 